\title{
SOME REMARKS ON DEFORMATIONS OF MINIMAL SURFACES
}

\author{
HAROLD ROSENBERG AND ERIC TOUBIANA
}

\begin{abstract}
We consider complete minimal surfaces (c.m.s.'s) in $R^{3}$ and their deformations. $M_{1}$ is an $\varepsilon$-deformation of $M_{0}$ if $M_{1}$ is a graph over $M_{0}$ in an $\varepsilon$ tubular neighborhood of $M_{0}$ and $M_{1}$ is $\varepsilon C^{1}$-close to $M_{0}$. A minimal surface $M$ is isolated if all c.m.s.'s which are sufficiently small deformations of $M$ are congruent to $M$.

In this paper we construct an example of a nonisolated c.m.s. It is modelled on a 4-punctured sphere and is of finite total curvature. On the other hand, we prove that a c.m.s. discovered by Meeks and Jorge, modelled on the sphere punctured at the fourth roots of unity, is isolated.
\end{abstract}

Introduction. We consider complete minimal surfaces (c.m.s.'s) in $R^{3}$ and their deformations. $M_{1}$ in an $\varepsilon$-deformation of $M_{0}$ if $M_{1}$ is a graph over $M_{0}$ is an $\varepsilon$ tubular neighborhood of $M_{1}$ and $M_{1}$ is $\varepsilon C^{1}$-close to $M_{0}$. A c.m.s. $M_{0}$ is isolated if all minimal surfaces $M_{1}$, which are sufficiently small deformations of $M_{0}$, are congruent to $M_{0}$. Many of the classical minimal surfaces in $R^{3}$ are known to be isolated [2], however, no example was known of a nonisolated minimal surface. In this paper we construct such an example; it is modelled on a 4-punctured sphere and is of finite total curvature. On the other hand, we prove that a c.m.s. discovered by Meeks and Jorge, modelled on the sphere punctured at the four roots of unity, $\{1,-1, i,-i\}$, is isolated. The analogous surface modelled on the sphere punctured at the cube roots of unity was shown to be isolated in [2]. The question is raised there of whether deformations of the four puncture case can be realized by deforming the conformal structure; i.e., changing the cross ratio of the four points. Thus for the Meeks-Jorge example in question, the answer is no. As we shall see, the conformal structure of our example that admits deformations does not change either. Perhaps the conformal structure never changes by small deformations? We wish to thank W. Meeks and B. Morin for helpful conversations and greatly simplifying suggestions.

I. A deformable surface. Let $M$ be a c.m.s. of finite total curvature, so that $M$ is conformally equivalent to a compact Riemann surface $\bar{M}$ punctured at a finite number of points. An end $E$ of $M$ is said to be bounded if $E$ is a bounded distance from a plane. If $E$ corresponds to the puncture $p$ and $P$ is the plane orthogonal to $g(p), g$ the Gauss map, then $E$ bounded means $E$ is a bounded distance from $P$. If $E$ is embedded, then it can be expressed as the graph of $a \log R+O(|R|)$, where $R$ is the distance from the origin in $P$, and this holds for $R$ large. Then $E$ is bounded if and only if $a=0$.

Let $(g, \omega)$ be a Weierstrass representation of $M$ and $\phi_{1}, \phi_{2}, \phi_{3}$ the associated analytic differentials on $M$. We know the real periods of each $\phi_{k}$ are zero on $M$

Received by the editors December 2, 1983 and, in revised form, May 1, 1984. 1980 Mathematics Subject Classification (1985 Revision). Primary 53A10. 
since the coordinate functions, $x_{k}=\operatorname{Re} \int \phi_{k}$, are well defined on $M$. Suppose in addition, that each $\phi_{k}$ has no imaginary periods on $M$. Let $\phi_{k}(t)=e^{i t} \phi_{k}$ for each $t \in R$. Then $\phi_{k}(t)$ are analytic differentials on $M$ which have no periods on $M$. Thus $x_{k}(t)=\operatorname{Re} \int \phi_{k}(t)$ are single-valued coordinate functions on $M$, giving a c.m.s. $M(t)$ modelled on $M$. We claim that if each end of $M$ is bounded, then $M(t)$ is an $\varepsilon$-deformation of $M$ for $t$ sufficiently small. We will prove this by showing that each end of $M(t)$ is a deformation of the corresponding end of $M(0)=M$; this suffices since the compact part of $M(t)$, the complement of the ends, converges uniformly to the compact part of $M$. Let the end $E$ of $M$ be parametrized by the disc unity $D$ punctured at 0 . Since deforming (i.e., $\left.\left(g, e^{i t} \omega\right)\right)$ commutes with rotation in $R^{3}$, we can assume $g(0)=0$. Then $g$ has a zero of order $n$ at 0 and $\omega$ has a pole of order $k \geq 2$. Clearly $E$ is a bounded end if and only if $n \geq k$ (look at $\left.x_{3}=\operatorname{Re} \int g \omega\right)$. Now $\left(g, e^{i t} \omega\right)$ is a Weierstrass representation of $M(t)$ and 0 is still a pole of order $k$ of $e^{i t} \omega$ so $E(t)$ is bounded as well. Thus $x_{3}(t)$ is bounded in the punctured disc, hence extends to the origin. Since

$$
x_{3}(t)=\cos (t) x_{3}(0)-\sin (t) \operatorname{Im} \int \phi_{3}
$$

we conclude $\operatorname{Im} \int \phi_{3}$ is bounded in the punctured disc. Thus $x_{3}(t)$ converges to $x_{3}(0)$ uniformly and it follows that $E(t)$ is a deformation of $E$.

Thus to exhibit a nonisolated c.m.s. we will find a c.m.s. $M$ of finite total curvature, with bounded ends, all periods of the $\phi_{k}$ are zero, and $M(t)$ is not congruent to $M$ for $t \neq 0$. Such an example follows.

Let $M$ be the sphere punctured at the four points $a_{1}, a_{2}, a_{3}, \infty$, where the $a_{k}$ are the cube roots of $-1 / 2$. Let

$$
g(z)=-\frac{1}{2} \frac{z}{\left(z^{3}-1\right)}, \quad \omega=\frac{1}{4} \frac{\left(z^{3}-1\right)^{2}}{\left(z^{3}+1 / 2\right)^{2}} d z .
$$

We claim $(g, \omega)$ is a Weierstrass representation of $M$ satisfying the conditions desired.

In fact, this example is part of the following family, all of which admit deformations:

$$
g(z)=-\frac{1}{2} \frac{z}{\left(z-b_{1}\right)\left(z-b_{2}\right)\left(z-b_{3}\right)}, \quad \omega=\frac{\prod_{i=1}^{3}\left(z-b_{i}\right)^{2}}{\prod_{i=1}^{3}\left(z-a_{i}\right)^{2}} d z
$$

where the $a$ 's and b's satisfy

$$
\begin{aligned}
& a_{i} \neq a_{j}, \quad b_{i} \neq b_{j}, \quad i \neq j \quad \text { and } a_{i} \neq b_{j}, \\
& b_{1}+b_{2}+b_{3}=0, \quad 2 a_{1}^{3}=-b_{1} b_{2} b_{3}, \\
& a_{2}=e^{2 \pi i / 3} a_{1}, \quad a_{3}=e^{4 \pi i / 3} a_{1} .
\end{aligned}
$$

We now prove that each of these surfaces is not isolated. First observe that

$$
g^{\prime}(z)=\pi\left(z-a_{i}\right) / \pi\left(z-b_{i}\right)^{2}
$$

and $d g$ vanishes at each pole of $\omega$, so each end is bounded (each pole is of order two). 
Next observe that $\phi_{1}, \phi_{2}, \phi_{3}$ have no periods; to see this it suffices that $\omega, g \omega$, and $g^{2} \omega$ be exact. We have

$$
\begin{aligned}
\omega & =d\left[\frac{z^{4}-2 b z^{2}-4 a_{1}^{3} z-b^{2} / 3}{a}\right] \\
g \omega & =\frac{1}{2} d\left[\frac{z^{2}+b / 3}{a}\right], \quad g^{2} \omega=-\frac{1}{12} d\left(\frac{1}{a}\right),
\end{aligned}
$$

where $b=b_{1} b_{2}+b_{1} b_{3}+b_{2} b_{3}$ and $a=\prod_{i=1}^{3}\left(z-a_{i}\right)$.

The reader can verify that this is a c.m.s. We will now prove the associate surface $M(t)=\left(g, e^{i t} \omega\right)$ is not congruent to $M$. Assume the contrary; for each $t$, let $M=R_{t}(M(t)+T(t))$, where $T(t)$ is a translation and $R_{t}$ is an affine rotation. The vectorial rotation defined by $R_{t}$ (that is, leaving the origin fixed) permutes the ends $\left\{0, g\left(a_{1}\right), g\left(a_{2}\right), g\left(a_{3}\right)\right\}$ since $M$ and $M(t)$ have the same limiting normals at the ends. There are only a finite number of such vectorial rotations so, for some sequence $t_{n} \rightarrow 0$, we have a rotation $R$ and translations $P_{n}$ such that

$$
M=\left(R P_{n}\right)\left(M\left(t_{n}\right)+T\left(t_{n}\right)\right)=R\left(M\left(t_{n}\right)+P_{n}+T\left(t_{n}\right)\right) .
$$

Therefore, the surfaces $M\left(t_{n}\right)+P_{n}+T\left(t_{n}\right)$ are the same for each $n$.

Now each of these surfaces has the same Gauss map $g$. The order of $g$ on $M$ is three and near each end the order is two $\left(g^{\prime}=0\right.$ and $g^{\prime \prime} \neq 0$ at each end). Thus for each end $p_{i}, i=1,2,3,4$, there is one and only one point $z_{i}$ in $M$ such that $g\left(z_{i}\right)=g\left(p_{i}\right), z_{i} \neq p_{i}$.

Let $K$ be a compact set in $M$ obtained by removing an open disc about each $p_{i}$ and with $z_{i} \in K$ for $i=1, \ldots, 4$. We know that on $K$, we can choose the parametrizations $X(t)$ of $M(t)$ close to a parametrization $X(0)=X$ of $M$ for $t$ sufficiently small, i.e., for $\varepsilon>0$ there exists $t>0$ such that for all $z \in K$, $d(X(z), X(t)(z))<\varepsilon$. Thus for $t, s$ close to 0 we have

$$
d(X(t)(z), X(s)(z)) \leq \varepsilon \quad \text { on } K .
$$

Let $p$ be an end and $z_{0} \in K$ such that $g\left(z_{0}\right)=g(p)$. We know that $M(t)$ and $M(s)$ differ by a translation $V(t, s)$ :

$$
M(t)=M(s)+V(t, s) .
$$

Hence there is $z_{0}^{\prime} \in M$ such that

$$
X(t)\left(z_{0}\right)=X(s)\left(z_{0}^{\prime}\right)+V(t, s) .
$$

The surfaces $M(t)$ and $M(s)$ have the same Gauss map so $g\left(z_{0}\right)=g\left(z_{0}^{\prime}\right)$. Thus $z_{0}^{\prime}=z_{0}$ or $z_{0}^{\prime \prime}=p$. But $X(s)\left(z_{0}^{\prime}\right) \in M(s)$ so $z_{0}^{\prime}=z_{0}$ and $X(t)\left(z_{0}\right)=X(s)\left(z_{0}\right)+$ $V(t, s)$. Then (I) implies $\|V(t, x)\| \leq \varepsilon$.

Now for $z$ in $K, z$ near $z_{0}$, the point $z^{\prime}$ such that $X(t)(z)=X(s)\left(z^{\prime}\right)+V(t, s)$ is near $z_{0}$ or near the end $p$. It is not hard to see it must be near $z_{0}$; we leave this to the reader. Since $g\left(z^{\prime}\right)=g(z)$, this implies $z^{\prime}=z$.

Thus $X(t)(z)=X(s)(z)+V(t, s)$ on an open set of $M$. By analytic continuation the equality holds on $M$. Then $\phi_{k}(t)=\phi_{k}(s), k=1,2,3$, and $\omega(t)=\omega(s)$. Hence $e^{i t} \omega=e^{i s} \omega$. As $t$ and $s$ are arbitrarily near 0 , we have $t=s=0$. This proves the surfaces $M$ are not congruent to their associate surfaces $M(t)$. 
II. An isolated surface. Let $M$ be the sphere punctured at the $n$ roots of unity. Meeks and Jorge have discovered examples of a c.m.s. modelled on $M$, of finite total curvature. Their Weierstrass representation is

$$
g(z)=z^{n-1}, \quad \omega=d z /\left(z^{n}-1\right)^{2} .
$$

When $n=2$ this gives the catenoid; in general this surface is rotationally symmetric and has $n$ catenoid type ends. In [2] we proved the surfaces $n=2$ and $n=3$ are isolated and the question was raised whether deformations for $n=4$ could be obtained by changing the conformal structure; i.e., moving the punctures to change the cross ratio. We shall now prove this is not possible. The technique is the same as that we used for $n=3$ and offers little hope of understanding $n>4$.

THEOREM 2.1. The c.m.s. $M_{0}$, modelled on the sphere punctured at the 4 roots of unity and defined by

$$
g_{0}(z)=z^{3}, \quad \omega_{0}=d z /\left(z^{4}-1\right)^{2},
$$

is isolated.

PrOOF. We proved in [2] that if $M_{0}$ is a c.m.s. conformally equivalent to a compact Riemann surface $\bar{M}$, punctured at $m$ points, and if $M_{1}$ is a deformation of $M_{0}$, then $M_{1}$ is conformally equivalent to $\bar{M}$ punctured at $m$ points (not necessarily the same points) and the total curvature of $M_{1}$ equals the total curvature of $M_{0}$.

Now suppose $M$ is a deformation of our example. Then $M$ is conformally equivalent to the sphere punctured at four points, and we can assume the points are $\left\{z_{0}, 1,-1, i\right\}$. Assume $z_{0} \neq \infty$; this case will be discussed later.

Let $(g, \omega)$ be a Weierstrass representation of $M ; g$ is a rational map of degree three since the total curvature of $M$ is that of $M_{0}$. Write

$$
g(z)=\frac{a z^{3}+b z^{2}+c z+d}{\alpha z^{3}+\beta z^{2}+\gamma z+\delta} .
$$

We assume $\delta \neq 0$; this case will be treated later. So normalize by $\delta=1$. We know $\omega$ has a double pole at the points $z_{0}, 1,-1, i$, the point $\infty$ is regular for $\omega$, and $\omega$ has a zero of order $2 k$ at each pole of $g$ of order $k$. We write

$$
\omega=\frac{e\left(\alpha z^{3}+\beta z^{2}+\gamma z+1\right)^{2}}{\left(z-z_{0}\right)^{2}(z-1)^{2}(z+1)^{2}(z-i)^{2}} d z
$$

We know the limiting normals of an end of $M_{0}$ and $M$ are the same, so $g(1)=1$, $g(-1)=-1, g(i)=-i, g\left(z_{0}\right)=i$. Write

$$
\frac{1}{\left(z-z_{0}\right)^{2}(z-1)^{2}(z+1)^{2}(z-i)^{2}}=\frac{A z+B}{\left(z-z_{0}\right)^{2}}+\frac{C z+D}{(z-1)^{2}}+\frac{E z+F}{(z+1)^{2}}+\frac{C z+H}{(z-i)^{2}} \text {. }
$$

Let $x_{k}, k=1,2,3$, be the coordinate functions of $M$ and $y_{k}$ those of $M_{0} ; x_{k}=$ $\operatorname{Re} \int \phi_{k}$ and $y_{k}=\operatorname{Re} \int \psi_{k}, \phi_{k}$ and $\psi_{k}$ are the differentials obtained via the Weierstrass representation. We know the real periods of the $\phi_{k}$ and $\psi_{k}$ are zero, since $x_{k}$ and $y_{k}$ are well defined functions on $M$ and $M_{0}$ respectively. From this we obtain three equations:

$$
\begin{gathered}
-G(-a i-b+c i+d)+(G i+H)[i(-3 \alpha+2 i \beta+\gamma)-(-3 a+2 b i+c)]=0 \\
-E(-a+b-c+d)+(F-E)[3 \alpha-2 \beta+\gamma-(3 a-2 b+c)]=0
\end{gathered}
$$


(3)

$$
c(a+b+c+d)+(C+D)(3 \alpha+2 \beta+\gamma+3 a+2 b+c)=0 .
$$

Equation (1) results from $\operatorname{Re}\left(i \operatorname{Res}\left(\phi_{1}, i\right)\right)=0$ and $\operatorname{Re}\left(-\operatorname{Res}\left(\phi_{3}, i\right)\right)=0$. Equation (2) follows from $\operatorname{Re}\left(i \operatorname{Res}\left(\phi_{2},-1\right)\right)=0$ and $\operatorname{Re}\left(i \operatorname{Res}\left(\phi_{3},-1\right)\right)=0$; equation (3) from $\operatorname{Re}\left(i \operatorname{Res}\left(\phi_{2}, 1\right)\right)=0=\operatorname{Re}\left(i \operatorname{Res}\left(\phi_{3}, 1\right)\right)$.

Now $g(1)=1$ so near the puncture $z=1$, the end of $M$ is a graph over the $\left(x_{2}, x_{3}\right)$ plane and a calculation yields

$$
x_{1}(z)=K \log \left|x_{3}+i x_{2}\right|+O\left(\left|x_{3}+i x_{2}\right|\right) \text { as } z \rightarrow 1 \text {, }
$$

where $K=-e(C+D)(a+b+c+d)[3 \alpha+2 \beta+\gamma-(3 a+2 b+c)]$. The same calculation for $M_{0}$ at this end yields

$$
y_{1}(z)=\frac{6}{32} \log \left|x_{3}+i x_{2}\right|+O\left(\left|x_{3}+i x_{2}\right|\right) \quad \text { as } z \rightarrow 1 \text {. }
$$

Since $M$ and $M_{0}$ are $\varepsilon$ close, the coefficients of $\log \left|x_{3}+i x_{2}\right|$ must be the same. This yields equation (4):

$$
-e(C+D)(a+b+c+d)[3 \alpha+2 \beta+\gamma-(3 a+2 b+c)]=6 / 32
$$

The same calculations at the punctures -1 and $i$ yield two more equations:

$$
-e(F-E)(-a+b-c+d)(3 \alpha-2 \beta+\gamma+3 a-2 b+c)=6 / 32,
$$

(6) $-e(G i+H)(-a i-b+c i+d)[i(-3 \alpha+2 \beta i+\gamma)+(-3 a+2 b i+c)]=6 i / 32$.

A calculation shows

$$
\begin{array}{ll}
C=\frac{(1-2 i)\left(1-z_{0}\right)-2 i}{8\left(1-z_{0}\right)^{3}}, & D=\frac{\left(1-z_{0}\right)(-1+3 i)+2 i}{8\left(1-z_{0}\right)^{3}}, \\
E=\frac{-(1+2 i)\left(1+z_{0}\right)-2 i}{8\left(1+z_{0}\right)^{3}}, & F=\frac{\left(1+z_{0}\right)(-1-3 i)-2 i}{8\left(1+z_{0}\right)^{3}}, \\
G=\frac{i\left(i-z_{0}\right)-1}{2\left(i-z_{0}\right)^{3}}, & H=\frac{3\left(i-z_{0}\right)+2 i}{4\left(i-z_{0}\right)^{3}}
\end{array}
$$

To the above six equations one must add the four equations $g(1)=1, g(-1)=-1$, $g(i)=-i, g\left(z_{0}\right)=i$. Now we have ten equations in nine unknowns. These equations have no solution. For completeness we show why this is so.

Replacing $C, D, E, F, G, H$ by their values and using the values of $g$ at the punctures, equations (1) through (6) become

$$
\begin{aligned}
& a\left(-3 z_{0}+i\right)+b\left(3 i z_{0}+1\right)+c\left(z_{0}+i\right)+d\left(i z_{0}+3\right)+2 z_{0}-2 i=0, \\
& a\left((1+3 i) z_{0}+1+i\right)+b\left((1-3 i) z_{0}+1-i\right)+c\left((-1+i) z_{0}-1-i\right) \\
& +d\left((1+i) z_{0}+1+3 i\right)+(-2-2 i) z_{0}-2-2 i=0, \\
& a\left((1-3 i) z_{0}-1+i\right)+b\left((-1-3 i) z_{0}+1+i\right)+c\left((-1-i) z_{0}+1-i\right) \\
& +d\left((-1+i) z_{0}+1-3 i\right)+(-2+2 i) z_{0}+2-2 i=0, \\
& a\left(2 z_{0}^{3}-i z_{0}^{2}-z_{0}\right)+b\left(-i z_{0}^{3}+z_{0}^{2}\right)+c\left(-i z_{0}^{2}+z_{0}\right) \\
& +d\left(-i z_{0}+1\right)-z_{0}^{3}+i z_{0}^{2}+z_{0}-i=0 \\
& \frac{e}{\left(1-z_{0}\right)^{2}}(a+b+c+d)((-1+2 i) a+b+c+d-2-2 i)=\frac{3}{2} i \text {, } \\
& \frac{e}{\left(1+z_{0}\right)^{2}}(-a+b-c+d)((1+2 i) a+b-c+d+2-2 i)=-\frac{3}{2} i, \\
& \frac{e}{\left(i-z_{0}\right)^{2}}(-a i-b+c i+d)(-a-i b-c+i d-2)=-\frac{3}{4} i \text {. }
\end{aligned}
$$


Equations (1), (2), (3) and $*$ give a linear system with four complex unknowns $a, b, c, d$ and one complex parameter $z_{0}$. When we resolve this system we get

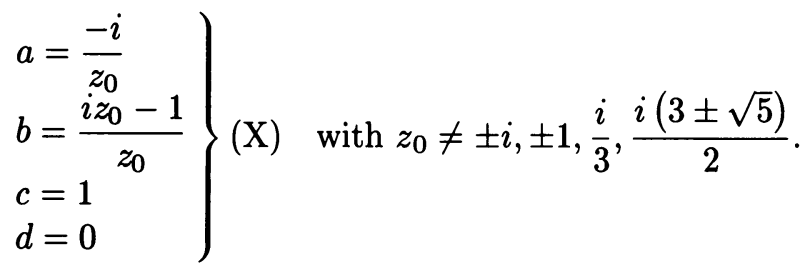

Suppose $z_{0}$ is not one of these complex numbers. Using (X), equation (6) becomes $0=-\frac{3}{4} i$. (This because $-a i-b+c i+d=\left(-1-\left(i z_{0}-1\right)+i z_{0}\right) / z_{0}=0$.) This shows that if $z_{0} \neq \pm i, \pm 1, i / 3, i(3 \pm \sqrt{5}) / 2$, then the equations (1)-(6), $*$ admit no solutions.

Now we must see what happens if the other case occurs. But $z_{0}$ cannot be $i, 1$ or -1 because $z_{0}$ is the fourth end of $M$. So we must only see $z_{0}=-i, i / 3, i(3 \pm \sqrt{5}) / 2$.

(1) Let us assume $z_{0}=-i$. Equations (1), (2), (3), * give

$$
\begin{aligned}
& a i+b+d-i=0, \\
& 2 a+b(-1-i)+d(1+i)-2=0, \\
& -2 a+b(-1+i)+d(1-i)+2=0, \\
& a=1 .
\end{aligned}
$$

We get $a=1, b=d=0$. Using that, equations (4) and (6) give

$$
\begin{aligned}
& e(c+1)(c-3)=-3, \\
& e(c-1)(c+3)=-3,
\end{aligned}
$$

and so $c=0$ and $e=1$. Thus we get finally if $z_{0}=-i, a=e=1 ; b=c=d=0$. This implies $\alpha=\beta=\gamma=0$. But this means that $g=g_{0}, \omega=\omega_{0}$ and then $M=M_{0}$.

(2) Let us assume $z_{0}=i / 3$. Equations (1), (2), (3), $*$ give

$$
\begin{aligned}
& c i+2 d-i=0 \\
& 2 a i+b(3-i)+c(-2-2 i)+d(1+5 i)-2-4 i=0 \\
& 2 a i+b(3+i)+c(2-2 i)+d(1-5 i)+2-4 i=0 \\
& -2 a i-b+3 i c+9 d-5 i=0 .
\end{aligned}
$$

This system gives: $a=-3 ; b=4 i ; c=1 ; d=0$. Using that and equation (6) we get a contradiction.

(3) The last case is $z_{0}=i(3 \pm \sqrt{5}) / 2$ or, equivalently: $z_{0}^{2}=3 i z_{0}+1$, and this implies $z_{0}^{3}=-8 z_{0}+3 i$. Using that, equation $*$ becomes

$$
a\left(-14 z_{0}+5 i\right)+b\left(11 i z_{0}+4\right)+c\left(4 z_{0}-i\right)+d\left(-i z_{0}+1\right)+6 z_{0}-3 i=0 .
$$

Equations (1), (2), (3) and (4) give: $a=-i / z_{0} ; b=i z_{0}-1 / z_{0} ; c=1 ; d=0$, with $z_{0}=i(3 \pm \sqrt{5}) / 2$. But we again get a contradiction to equation (6).

So if $z_{0} \neq \infty$ and $\delta \neq 0$, the only solution is $z_{0}=-i ; a=e=1 ; b=c=d=$ $\alpha=\beta=\gamma=0$; this means $g=g_{0}, \omega=\omega_{0}$ and so $M=M_{0}$. 
Let us assume now $z_{0}=\infty . M$ is then modelled on the sphere punctured at $\infty$, $1,-1, i$. The homography $T$ of $S^{2}, T(z)=-1 / z$, maps $(\infty, 1,-1, i)$ to $(0,-1,1, i)$ respectively. Thus we can assume $M$ is modelled on $S^{2}-\{0,-1,1, i\}$.

The Weierstrass representation of $M$ is

$$
g(z)=\frac{a z^{3}+b z^{2}+c z+d}{\alpha z^{3}+\beta z^{2}+\gamma z+1}, \quad \omega=\frac{e\left(\alpha z^{3}+\beta z^{2}+\gamma z+1\right)^{2}}{z^{2}(z+1)^{2}(z-1)^{2}(z-i)^{2}} d z .
$$

As $M$ is $C^{1}$-close to $M_{0}$, we must have

$$
\begin{aligned}
& g(0)=g_{0}(-i)=i, \quad g(-1)=g_{0}(1)=1, \\
& g(1)=g_{0}(-1)=-1, \quad g(i)=g_{0}(i)=-i \text {. }
\end{aligned}
$$

This gives

$$
\begin{aligned}
& d=1 \\
& -a+b-c+d=-\alpha+\beta-\gamma+1, \\
& a+b-c+d=-\alpha-\beta-\gamma-1, \\
& -a i-b+c i+d=-\alpha+\beta i+\gamma-i .
\end{aligned}
$$

At last

$$
\begin{aligned}
& d=i, \quad \alpha=-i c-2 i, \\
& \beta=-a-c-1, \quad \gamma=-b+i c+i .
\end{aligned}
$$

In the same way as before (i.e., $z_{0} \neq \infty$ ), we get six equations:

(3) $-G(-a i-b+c i+i)+(G i+H)(i(-3 \alpha+2 \beta i+\gamma)-(-3 a+2 b i+c))=0$,

(6) $e i(G i+H)(-a i-b+c i+i)(i(-3 \alpha+2 \beta i+\gamma)+(-3 a+2 b i+c))=6 / 32$, with

$$
\begin{cases}C=\frac{1-4 i}{8}, & D=\frac{-1+5 i}{8}, \\ E=\frac{-1-4 i}{8}, & F=\frac{-1-5 i}{8}, \\ G=-i, & H=-\frac{5}{4} .\end{cases}
$$

Using (C) and (D), equations (1) and (2) become

$$
\begin{aligned}
& a(1+i)+b(1-i)+c(-1-i)-1+3 i=0, \\
& a(1-i)+b(-1-i)+c(-1+i)-1-3 i=0 .
\end{aligned}
$$

But

$$
\text { (1) }+ \text { (2) } \Rightarrow a-b i-c-1=0 \text {, }
$$




$$
\text { (1) }-(2) \Rightarrow a i+b-c i+3 i=0
$$

and

$$
\text { (1) }+i \times(2) \Rightarrow 4=0 \text {. }
$$

This shows that equations (1)-6) admit no solution, so $z_{0}$ cannot be $\infty$.

At last, let us assume $\delta=0$. Then,

$$
g(z)=\frac{a z^{3}+b z^{2}+c z+d}{\alpha z^{3}+\beta z^{2}+\gamma z}, \quad \omega=\frac{e\left(\alpha z^{3}+\beta z^{2}+\gamma z\right)^{2}}{\left(z-z_{0}\right)^{2}(z-1)^{2}(z+1)^{2}(z-i)^{2}} d z .
$$

We have $g(1)=1, g(-1)=-1, g(i)=-i, g\left(z_{0}\right)=i$.

$$
\begin{aligned}
& g(1)=1 \Leftrightarrow a+b+c+d=\alpha+\beta+\gamma, \\
& g(-1)=-1 \Leftrightarrow-a+b-c+d=\alpha-\beta+\gamma, \\
& g(i)=-i \Leftrightarrow-a i-b+c i+d=-\alpha+\beta i+\gamma, \\
& g\left(z_{0}\right)=i \Leftrightarrow a z_{0}^{3}+b z_{0}^{2}+c z_{0}+d=i \alpha z_{0}^{3}+i \beta z_{0}^{2}+i \gamma z_{0} .
\end{aligned}
$$

These equations yield

(E) $\left\{\begin{array}{l}\alpha=a i+b, \quad \beta=a+c, \quad \gamma=-a i+d, \\ \circledast a\left(2 z_{0}^{3}-i z_{0}^{2}-z_{0}\right)+b\left(-z_{0}^{3}+z_{0}^{2}\right)+c\left(-i z_{0}^{2}+z_{0}\right)+d\left(-i z_{0}+1\right)=0 .\end{array}\right.$

Using $(\mathrm{E})$ and $\operatorname{Re}\left[i \operatorname{Res}\left(\phi_{j}, \theta\right)\right]=0, j=1,2,3$, where $\theta=1,-1, i$, we get three equations:

$$
\begin{aligned}
& a\left(-3 z_{0}+i\right)+b\left(3 i z_{0}+1\right)+c\left(z_{0}+i\right)+d\left(i z_{0}+3\right)=0, \\
& a\left(z_{0}(1+3 i)+1+i\right)+b\left(z_{0}(1-3 i)+1-i\right)+c\left(z_{0}(-1+i)-1-i\right) \\
& +d\left(z_{0}(1+i)+1+3 i\right)=0 \\
& \text { (3) } \quad a\left(z_{0}(1-3 i)-1+i\right)+b\left(z_{0}(-1-3 i)+1+i\right)+c\left(z_{0}(-1-i)+1-i\right) \\
& +d\left(z_{0}(-1+i)+1-3 i\right)=0 \text {. }
\end{aligned}
$$

With $\circledast$, we get a linear system with four complex unknowns, $a, b, c$ and $d$. The determinant $D$ is

$$
D=-8 i z_{0}\left(z_{0}-1\right)\left(z_{0}+1\right)\left(z_{0}+i\right)\left(z_{0}-i\right)^{2} .
$$

Thus, if $D \neq 0$ the only solution is $a=b=c=d=0$, but this means $g=0$ and $M$ is part of a plane and then $M$ cannot be $C^{1}$-close to $M_{0}$.

Let us assume $D=0$. Here $D=0$ only if $z_{0}=0$ or $z_{0}=-i$.

(1) $z_{0}=0$. But $* \Rightarrow d=0$ and this implies that the order of $g$ is 2 and not 3 because

$$
g(z)=\frac{a z^{3}+b z^{2}+c z}{\alpha z^{3}+\beta z^{2}+\gamma z}=\frac{a z^{2}+b z+c}{\alpha z^{2}+\beta z+\gamma} .
$$

(2) $z_{0}=-i$. Equations (1), (2), (3) and $*$ become

$$
\begin{aligned}
& a i+b+d=0, \\
& 2 a-b(1+i)+d(1+i)=0, \\
& -2 a-b(1-i)+d(1-i)=0, \\
& a=0 .
\end{aligned}
$$

This gives $a=b=d=0$, but $d=0$ is not allowed as in the case $z_{0}=0$.

Thus $\delta$ cannot be zero and $M_{0}$ is then isolated. 


\section{BIBLIOGRAPHY}

1. W. Meeks, A survey of the geometric results in the classical theory of minimal surfaces, Bol. Soc. Brasil. Mat. 12 (1981), 29-86.

2. H. Rosenberg, Deformations of complete minimal surfaces, Trans. Amer. Math. Soc. 295 (1986), 475-489.

Département de Mathématiques, Université Paris VII, 2 Place Jussieu, 75005 PARIS, FRANCE 